\title{
Investigation of mode III fatigue crack growth in rubber based on the pioneering work of Alan N. Gent and coworkers
}

\author{
Quentin Demassieux ${ }^{\mathrm{a}, \mathrm{b}}$, Bertrand Huneau ${ }^{\mathrm{a}}$, Erwan Verron ${ }^{\mathrm{a}, *}$, Daniel Berghezan ${ }^{\mathrm{b}}$ \\ a Institut de Recherche en Génie Civil et Mécanique (GeM), UMR CNRS 6183, École Centrale de Nantes, France \\ ${ }^{\mathrm{b}}$ Michelin, Centre Technique de Ladoux, Clermont-Ferrand, France
}

\begin{abstract}
From 1998 to 2003, Alan N. Gent and some co-workers published a series of papers devoted to the first measurements of fatigue crack growth properties in rubber in mode III loading conditions (Rubber Chemistry and Technology, vol. 71, pp. 76-83; vol. 71, pp. 84-94; vol. 76, pp. 1276-1289). The present paper proposes to revisit this seminal work benefiting from the capabilities of new multiaxial fatigue machines. Then, a new mode III fatigue test for elastomers is developed. A thin bonded disk of rubber with a circumferential cut is subjected to cyclic torsion to investigate the fatigue crack growth in mode III. The corresponding measurement methods for the crack growth rate and the fracture energy are proposed. As a constant small axial extension can be applied and controlled to prevent the cracking surfaces from abrasion and to ensure almost mode III loading conditions, the quality and the relevance of the experimental data are highly enhanced. As a results, the method is applied to an unfilled natural rubber and the crack growth rate is related to the fracture energy for several loading cases. The effects of axial extension, alternate cycles and positive minimum angle cycles are examined. In particular, it is shown that for fully unloaded cycles the crack growth rate laws in modes I and III coincide in the regime of high fracture energies.
\end{abstract}

\author{
Keywords: \\ Fatigue \\ Mode III \\ Crack growth rate
}

\section{Introduction}

Industrial rubber parts often admit complex shape and are subjected to multiaxial loading conditions. As an example, tires experience tension induced by pressure, and shear and compression induced by contact with the road. Then, these complex loading conditions can induce any type of crack opening, i.e. modes I, II and III [1].

On the one hand, fatigue of rubber in mode I has been largely studied with tension and pure shear experiments, for which numerous experimental data are available, see for example [2,3]. On the other hand, only very few works have been proposed in mode III. For quasi-static loading conditions, Gdoutos et al. considered trousers samples [4,5], and Cha and Jeong performed peeling experiments [6]. For fatigue loading conditions, crack growth in mode III was studied in a series of papers due to Gent and coworkers [7-9]. In this study, the authors have (I) developed a dedicated mechanical system that was adapted on a classical tensile machine to prescribe torsion loading conditions [8], (II) derived the equations that permit to analyze experimental measurements [7], and (III) improved their results with the help of finite element analysis [9]. Recently, Keller et al. used the same kind of device to perform mode III fatigue test on self-healing elastomers [10].

The aim of the present paper is to revisit the experiments of Gent and co-workers with the help of new commercial fatigue tension/torsion machines devoted to soft materials. ${ }^{1}$ Indeed, we propose here a specific sample and the corresponding testing methodology to investigate the crack growth in mode III fatigue for rubber. The emphasis is laid on the thorough control of the loading conditions that permits us to overcome the difficulties encountered in [8]: the small mode I opening of the crack is controlled and permits us to substantially limit abrasion of cracked surfaces. The outline of this paper is as follows. First, Section 2 presents the sample, the machine and the methods to measure the crack growth rate $d c / d n$ (with $c$ being the crack length and $n$ being the number of cycles) and the fracture (or tearing) energy $G$. Then, Section 3 presents and discusses the results: pure mode III measurements are compared with those previously published, and as suggested in the fourth conclusion of [9] new loading

\footnotetext{
${ }^{1}$ Throughout the paper, notations and terminology used in the papers of Gent and coworkers are adopted.
} 


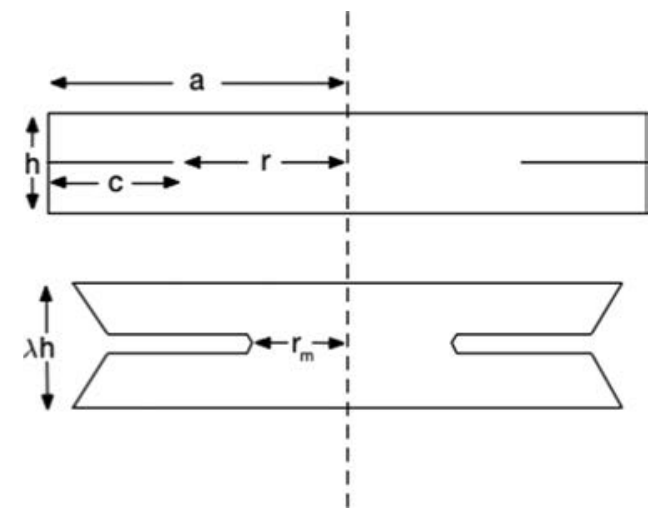

Fig. 1. Sample. Top: reference configuration. Bottom: deformed configuration.

conditions, i.e. superimposed modes I and III, and alternate fatigue, are investigated. Finally, a short conclusion closes the paper.

\section{Methods}

\subsection{Sample}

\subsubsection{Geometry}

The sample is a thin cylinder with a circumferential cut. In the undeformed configuration, its height is $h=5.7 \mathrm{~mm}$ and its radius is $a=30 \mathrm{~mm}$. These dimensions are almost the same as those considered in [8]. The crack depth in the undeformed state is denoted as $c$. We note $c^{0}$ the particular value of $c$ at the beginning of the experiment. The uncracked portion of the radius is $r=a-c$. Under simultaneous tension and torsion, the cylinder deforms and the crack grows; the corresponding deformed height and radius are respectively $\lambda h$ and $r_{m}$ where $\lambda$ is the axial stretch ratio and the subscript ${ }_{m} m$ stands for "measured". The geometry of the sample is sketched in Fig. 1.

\subsubsection{Fabrication}

First, raw unfilled rubber is laminated into thin sheets from which circular parts are punched with a die cutter to form small cylinders of about $30 \mathrm{~mm}$ in radius and $7-8 \mathrm{~mm}$ in height. Then, the surface of steel-end pieces is prepared for gluing: they are roughened and a Chemosyl adhesive, a strong rubber-metal adhesive that polymerizes during the molding process, is applied. Samples are formed in a three-part steel mold. The mold is preheated at $150{ }^{\circ} \mathrm{C}$ and the forming pressure is set at about $10 \mathrm{MPa}$. Both metal end-pieces and raw rubber are introduced in the mold. Once molding is completed, samples are extracted and left for cooling at room temperature. Finally, dimensions of the samples are verified; they almost always satisfy the required design. Nevertheless, due to the manual preparation of raw rubber, a given sample can exhibit a $0.1 \mathrm{~mm}$ variation in height.

The second step of fabrication is the cutting of the initial crack. It is to note that this step must be carefully performed to ensure a homogeneous initial crack depth $c$ in the samples and the parallelism of the crack faces with the surface of the steel-end pieces. Our method consists in using a utility knife mounted on a rigid support: the uncracked sample is placed in a lathe and rotation is prescribed, then the blade is slowly and carefully moved to cut it in the middle plane.

\subsection{Machine and loading conditions}

The above-described samples have been designed for the Instron ElectroPuls ${ }^{\mathrm{TM}} \mathrm{E} 10000$ uniaxial tension/torsion fatigue machine shown in Fig. 2. This machine permits us to prescribe

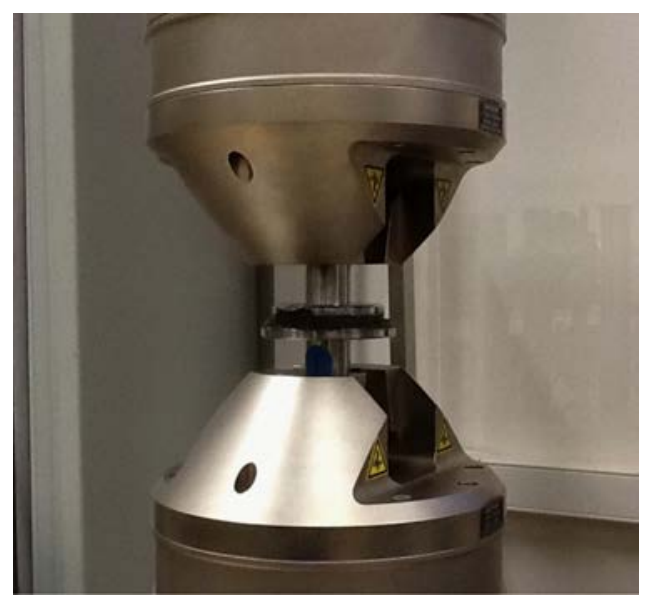

Fig. 2. A sample in the fatigue machine.

the axial displacement ${ }^{2} d$ or the axial force $F$, and the angle of twist $\phi$ or the torque $M$. In fact, the major difference with the experimental work of Gent and co-workers is the possibility to control the axial displacement or force and then to limit rubbing of cracked surfaces. Indeed, the experiments conducted in [8] correspond to $F=0$ in the present case. Here, we investigated the influence of $d$ and $F$ on the measurements, and we empirically concluded that $d=0.5 \mathrm{~mm}$ is a good compromise to limit rubbing and to minimize the influence of mode I loading conditions.

\subsection{Methods of measurements}

Classical results of fatigue crack growth experiments are presented as $\log (d c / d n)$ (fatigue crack growth rate) vs. $\log G$ (fracture energy) curves. Indeed, drawing these types of curves necessitates rigorous methods to measure $c, d c / d n$ and $G$.

\subsubsection{Crack depth}

In order to determine the crack depth $c$, the crack radius $r$ must be measured. To measure it in real-time during the test and to ensure a sufficient accuracy, two different methods have been considered: a "direct" method that gives $r$ at given number of cycles, and a "deduced" method, similar to the method adopted in [8], that uses $F$ and $M$ to deduce $r$ in real-time. Practically, the former method permits us to validate the latter one.

Direct method: During each fatigue test, at least four measurements of $r$ are performed: the initial crack depth (after cutting of the sample), the final crack depth (measured on the fracture surface after sufficient crack growth and cutting of the sample), and two or more intermediate values.

Practically, the test is stopped at the maximum strain of a cycle, the crack is open by the axial tensile force and the uncracked deformed radius $r_{m}$ is measured with an inextensible string of length $L$. The string is tied around the uncracked part of the sample, and its dangling length $l_{d}$ is measured by a vernier caliper; it leads to $r_{m}=l / 2 \pi$ with $l=L-l_{d}$. In order to obtain the uncracked undeformed radius $r$, a simple linear equation is used. At the beginning of the experiment, $c^{0}$ and then $r^{0}=a-c^{0}$ are known, and $r_{m}^{0}$ is measured under tension. Similarly, the final deformed crack depth $r_{m}^{f}$ is measured under tension, and the undeformed final crack depth $r^{f}$ is measured on the final cut surface. Thus,

$r \approx r^{0}+\frac{r_{m}-r_{m}^{0}}{r_{m}^{f}-r_{m}^{0}}\left(r^{f}-r^{0}\right)$.

\footnotetext{
${ }^{2}$ The stretch ratio is related to the axial displacement by $\lambda=1+d / h$.
} 


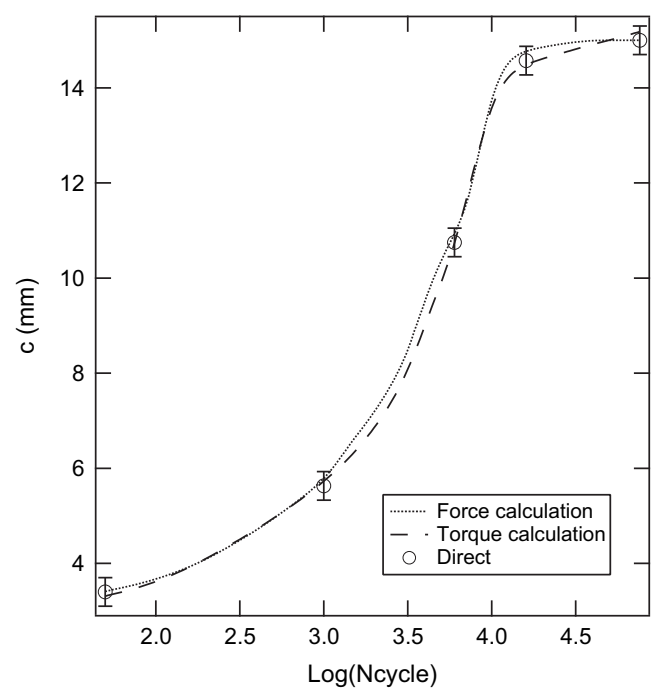

Fig. 3. Comparison of measurement methods for the crack depth.

Deduced method: The second method consists in relating the evolution of $r$ to the ones of $F$ and/or $M$. As the force and the torque can be recorded (or prescribed) throughout the cycles, it leads to the continuous measurement of the uncracked radius with time. As emphasized in [7], for pure torsion and simple constitutive equations the torsional stiffness, i.e. $M / \phi$, is a function of $r^{4}$. Motivated by this result, we simply propose to relate $r$ to $F$ and $M$ by power laws which empirically account for the coupling between modes I and III, and the complex non-linear mechanical response of rubber materials:

$r \approx A F^{\alpha}$ and $r \approx B M^{\beta}$

where $A, B, \alpha, \beta$ are scalar parameters that depend on a given sample. Practically, these parameters are identified with the values at the beginning and at the end of the experiments, i.e. $r^{0}, T^{0}, M^{0}$ and $r^{f}, T^{f}, M^{f}$ respectively.

In order to illustrate the relevance of the deduced method, Fig. 3 presents the evolution of the crack depth $c$ as a function of the number of cycles for a given sample and arbitrary loading conditions, as obtained by the three methods: by direct measurement, as a deduced function of the force $F$ and as a deduced function of the torque $M$. As shown in this figure, the data deduced from the force and the torque lead to almost the same curves, and they are validated by the three direct measurements depicted in the figure.

\subsubsection{Crack growth rate}

Once the crack depth is measured, the next step consists in determining the fatigue crack growth rate $d c / d n$ (in meter per cycle). By nature, fatigue experiments lead to scattered results. Then, in order to quantify the scattering of our results in terms of crack growth rate, we must analyze the observational error in the measurements of $c$. It is due to both the resolution error on measured data and the interpolation systematic error induced by the above-mentioned "deduced" method. First, the resolution error on the measurement of $r_{m}$ is

$\Delta r_{m}=\frac{\Delta l}{2 \pi}$

The resolution of the vernier caliper is equal to $0.1 \mathrm{~mm}$, but considering the possible (even limited) extensibility of the string and the possible human error, we estimate the resolution error as
$\Delta l=2.0 \mathrm{~mm}$. Thus,

$\Delta r_{m} \approx 0.3 \mathrm{~mm}$.

Concerning the interpolation error, we observe in Fig. 3 that the maximum distance between the two interpolated curves (force and torque) and the direct measurements (unfilled circles) does not exceed $0.3 \mathrm{~mm}$. Thus, the total observational error in the measurement of $c$ is less than $0.6 \mathrm{~mm}$. Obviously, the error on $d c / d n$ cannot be directly deduced from the error on $c$. However, as a systematic error on $c$ is nullified by differentiation and as the random noise is minimized by averaging between two data points, the error on $d c / d n$ should not exceed the error usually observed in mode I experiments, i.e. around half a decade.

\subsubsection{Fracture energy $G$}

Following Rivlin and Thomas, the fracture energy, also known as the tearing energy in rubber science or the energy release rate in the classical theory of fracture mechanics, is defined as [11]

$G=-\left.\frac{\partial U}{\partial A}\right|_{d, \phi}$

where $U$ is the total strain energy in the sample, $A$ is the cracked surface, and the subscript $\left.\cdot\right|_{d, \phi}$ means that the differentiation is made at constant displacement, i.e. external forces do no work.

Analytical solution: In their seminal paper, Rivlin and Thomas proposed analytical formulae for different samples used for crack growth in rubber. Nevertheless, the corresponding formula for the present sample has been proposed in [7]; the authors consider that the sample can be seen as an infinitely long neo-Hookean cylinder of radius $r$ subjected to pure torsion, i.e. the cracked portion of the disk is assumed to be stress-free and to carry no load. Thus, the corresponding strain energy reduces to

$U=\mu \frac{\pi \phi^{2}}{4 h} r^{4}$

where $\mu$ is the shear modulus. Then, invoking Eq. (5) and noting that the cracked area is $A=\pi\left(a^{2}-r^{2}\right)$, the fracture energy is

$G=\mu \frac{\phi^{2}}{2 h} r^{2}$.

Moreover, in the same paper, the authors derive a corrected formula to relax the previous assumption: in order to account for the area near the crack front that is not completely unloaded, they introduced a small correction factor $\delta$ such that

$G=\mu \frac{\phi^{2}}{2 h} r^{2}\left(1+\frac{\delta}{r}\right)^{3}$

Finally, they empirically set $\delta=0.22 h$ for their subsequent calculations.

In our experiments, the axial extension of the sample can be prescribed; then this must be taken into account in the derivation of the fracture energy. Similarly in the basic formula Eq. (7), we consider a neo-Hookean cylinder of radius $r$ subjected to simultaneous uniaxial extension $\lambda$ and torsion $\phi$. In a system of cylindrical coordinates $(\rho, \theta, z)$ with $z$ the axial direction, the left CauchyGreen tensor is given by [12]

$$
\begin{aligned}
\mathbf{B}= & \frac{1}{\lambda} \mathbf{e}_{\rho} \otimes \mathbf{e}_{\rho}+\frac{1}{\lambda}\left(1+K^{2}(\rho)\right) \mathbf{e}_{\theta} \otimes \mathbf{e}_{\theta} \\
& +\sqrt{\lambda} K(\rho)\left(\mathbf{e}_{\theta} \otimes \mathbf{e}_{z}+\mathbf{e}_{z} \otimes \mathbf{e}_{\theta}\right)+\lambda^{2} \mathbf{e}_{z} \otimes \mathbf{e}_{z}
\end{aligned}
$$

where $K(\rho)=\phi \rho / h$ and $\rho \in[0, r]$.

Then, the strain energy density in a given point is

$\left.W=\frac{\mu}{2} \frac{2}{\lambda}+\frac{K^{2}(\rho)}{\lambda}+\lambda^{2}-3\right)$ 
and by integration over the cylinder of radius $r$ and height $h$, it leads to

$U^{*}=\frac{\mu}{2} \pi r^{2} h\left(\frac{2}{\lambda}+\lambda^{2}-3\right)+\frac{w}{\lambda}$

where the second term in the right-hand side is simply the result of Aboutorabi et al. divided by the axial extension $\lambda$ [7]. With this strain energy, the fracture energy reduces to

$G^{*}=\frac{\mu}{2} h\left(\frac{2}{\lambda}+\lambda^{2}-3\right)+\frac{G}{\lambda}$,

where $G$ is given by Eq. (7). Indeed, the fracture energy is the sum of a contribution that corresponds to the uniaxial part of the loading conditions, i.e. the mode I,

$G_{I}=\frac{\mu}{2} h\left(\frac{2}{\lambda}+\lambda^{2}-3\right)$

and a contribution of the mode III loading conditions corrected by tension

$G_{I I I}=\frac{G}{\lambda}$.

In fact, with Eq. (12) it is possible to manage the relative contribution of mode I in the experiments. In the present work, $\lambda$ has been chosen such that the ratio $G_{I} / G^{*}$ did never exceed $4 \%$. Indeed, with such small values it is possible to conduct almost pure mode III fatigue tests with a substantial limitation of crack face abrasion.

Numerical validation: In the third paper of the series, published five years later than the first two ones, Gent and Yeoh investigated deviations from their simple analytical model with the help of Finite Element Analysis (FEA): they investigated the influence of initial crack depth and position on $G$ [9]. Here, we considered FEA to validate our previous results Eqs. (7) and (12). The commercial software Abaqus is used. With the help of the twist deformation degrees of freedom implemented in this software, and due to the geometrical symmetry of the sample, an axisymmetric model is adopted. Moreover, accounting for the symmetry with respect to the crack plane, only half of the sample is considered. So, quadratic elements with twist deformation and reduced integration are used. The main drawback of this type of finite elements is the impossibility to directly compute the fracture energy through a $J$-integral in the software. To overcome this difficulty, the method of global strain energy is adopted [9]: $G$ is calculated as the difference of the stored energy between two samples with slightly different crack depth, i.e. a discrete version of Eq. (5):

$G \approx-\frac{U(r+d r)-U(r)}{\pi\left((r+d r)^{2}-r^{2}\right)}$

Remark 1. In order to determine the mesh size and a relevant value for $d r$ in Eq. (15), a convergence study has been conducted. It has been shown that $d r$ must be higher than the element size, and that even a relatively coarse mesh is able to capture the right value of $G$ if $d r$ is well-chosen. Practically, for the present sample geometry (see Section 2.1.1) the mesh size is set at $0.25 \mathrm{~mm}$ and the corresponding mesh contains 1320 elements; then, $G$ is determined by adopting values of $d r$ between 0.5 and $1 \mathrm{~mm}$ depending on the loading conditions.

The major difficulty with this finite element model is the determination of the boundary conditions. The two extreme choices consist in fully constraining the radial displacement of the lower and upper boundaries, or letting it to be completely free. The former choice corresponds to the boundary conditions that apply on the real sample, the latter one corresponds to the analytical solution developed in [8]. In order to analyze the influence of this choice on $G^{*}$, we have compared them for pure torsion with the analytical solution

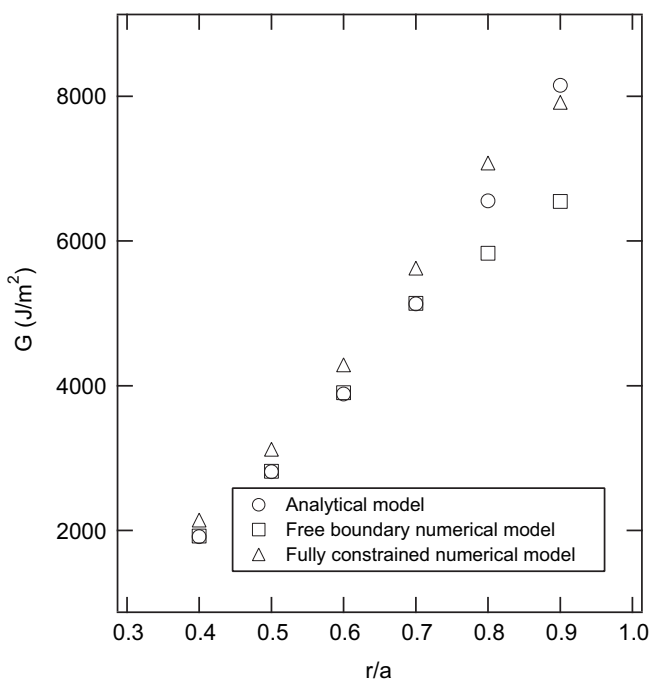

Fig. 4. Influence of the boundary conditions on $G^{*}$.

Table 1

NR composition. Data are given in phr (parts per hundred of rubber).

\begin{tabular}{lllllll}
\hline Compound & NR & Stearic acid & ZnO & CBS & Sulfur & 6PPD \\
\hline $\mathrm{phr}$ & 100 & 2 & 2.5 & 1.6 & 1.6 & 1.9 \\
\hline
\end{tabular}

$(\lambda=1$ in Eq. (12)). The corresponding results are presented in Fig. 4 for different values of the (relative) uncracked radius $r / a$. A similar analysis was proposed in the third paper of the series [9]. Depending on the shape ratio $r / h$, the analytical solution fits with the free radial displacement boundary conditions case for small ratios, i.e. $r / h \approx 2-3.7$, and tends to the case of fully constrained radial displacement for high ratios, i.e. $r / h \approx 5$. Then, this analysis first highlights that the analytical solution of the infinitely long cylinder remains valid even for short cylinders, and second shows that it underestimates $G^{*}$ by about $10 \%$ for low $r / h$ ratios, and overestimates $G^{*}$ for large $r / h$ ratios.

To conclude, the present numerical model demonstrates that the analytical solution for the fracture energy $G$ and, then $G^{*}$, can be used if we consider that the difference between the real solution, i.e. fully constrained radial displacement boundary condition, is not so large as compared with the scattering of fatigue measurements. Nevertheless, as we were able to develop a quite simple numerical model, i.e. in particular with an axisymmetric coarse mesh, which is not highly time consuming, all the values of $G$ and $G^{*}$ reported in the following have been obtained numerically.

\section{Results and discussion}

In this section, the previous methodology is applied to investigate the fatigue crack growth in mode III of natural rubber under various loading conditions.

\subsection{Materials and loading conditions}

The material studied is an unfilled natural rubber (NR) vulcanized during the formation of the sample. Its chemical composition is given in Table 1. As the maximum global extension prescribed to samples does not exceed $200 \%$, the material response is modeled by an isotropic incompressible hyperelastic neo-Hookean constitutive 
equation [13]. The corresponding shear modulus was determined with uniaxial tensile experiments: $\mu=0.44 \mathrm{MPa}$.

The mode III loading conditions are torsion cycles with prescribed angles; a typical experiment consists in about 80,000 cycles without failure. These loading conditions can be classified into three types:

- fully unloaded tests, during which the minimum torsional angle in a cycle is zero; these experiments are similar to the ones investigated by Gent and coworkers,

- partially unloaded tests, during which the minimum torsional angle remains positive,

- and finally alternate tests, during which the minimum torsional angle is the opposite of the maximum one.

As previously emphasized, all tests are conducted with a small tensile stretch to prevent abrasion of crack surfaces. Moreover,

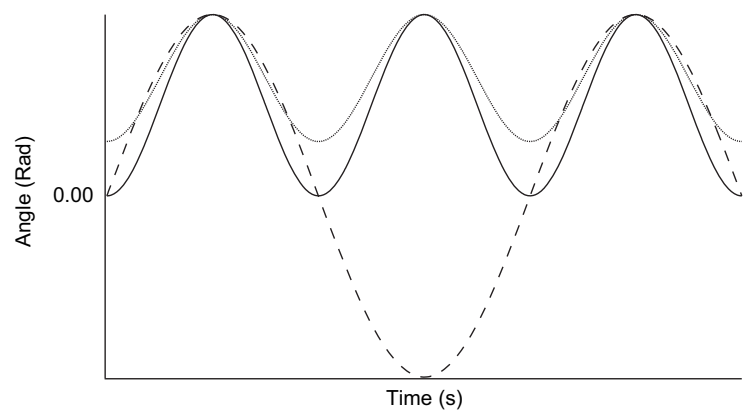

Fig. 5. Types of loading conditions: (-) fully unloaded test, $(\cdots)$ partially unloaded test, (- - -) alternate test.

Table 2

Loading conditions.

\begin{tabular}{llcll}
\hline Type & Sample & $\begin{array}{l}\text { Minimum } \\
\text { angle (deg) }\end{array}$ & $\begin{array}{l}\text { Maximum } \\
\text { angle (deg) }\end{array}$ & $\begin{array}{l}\text { Axial } \\
\text { displacement } \\
(\mathrm{mm})\end{array}$ \\
\hline \multirow{5}{*}{ Fully unloaded } & FU1 & 0 & 30 & 0.5 \\
& FU2 & 0 & 30 & 0.5 \\
& FU3 & 0 & 30 & 0.3 \\
Partially unloaded & FU4 & 0 & 30 & 1 \\
& PU1 & 5 & 30 & 0.5 \\
Alternate & PU3 & 7.5 & 30 & 0.5 \\
& A1 & -30 & 30 & 0.5 \\
& A2 & -30 & 30 & 0.5 \\
\hline
\end{tabular}

note that results obtained with the two latter types of experiments have never been published. These three types of tests are depicted in Fig. 5. Practically, nine different tests have been conducted. In order to consider an almost constant strain rate, the frequency is set to $1 \mathrm{~Hz}$ for fully and partially unloaded experiments, and to $0.5 \mathrm{~Hz}$ for alternate experiments. The corresponding loading conditions are given in Table 2 .

\subsection{Remark about abrasion of crack faces}

As already mentioned, the use of modern uniaxial tension/ torsion loading machine permits the application and control of a slight uniaxial extension in order to prevent the abrasion of cracked surfaces. This axial displacement leads to a mode I crack opening. As mentioned in Table 2, three values of the axial displacement were considered: $0.3,0.5$ and $1 \mathrm{~mm}$. In order to compare the efficiency of this method, the corresponding fracture surfaces, sliced after the end of the tests, are shown in Fig. 6; these photographs can be compared with the one proposed in Fig. 5 in [8] which exhibits abrasion patterns. In the three pictures, both the initial cut and the uncracked area can be distinguished; the crack growth zone takes place between these two zones. The crack growth zone highly differs depending on the axial displacement.

The sample with the smallest uniaxial opening (FU3, left hand side photograph in Fig. 6) is considerably abraded by rubbing: the crack growth zone is rough and the corresponding abrasion ridges are about $1 \mathrm{~mm}$ thick. Increasing the crack opening substantially reduces the roughness: for an axial displacement equal to $0.5 \mathrm{~mm}$, the thickness of the ridges is about $0.3 \mathrm{~mm}$ (see the middle photograph in Fig. 6). Finally, for the largest crack opening, i.e. $1 \mathrm{~mm}$ axial displacement, the fracture surface does not exhibit a ribbed surface as shown in the right-hand side photograph of Fig. 6. So, the mode I crack opening is efficient to limit surface abrasion during mode III experiments. Nevertheless, to conduct almost pure mode III experiments, a compromise must be reached between completely avoiding abrasion and limiting the influence of mode I loading conditions. In the present work, we chose to set the axial displacement to $0.5 \mathrm{~mm}$.

Finally, as easily seen in the three photographs of Fig. 6 small straight cracks take place in the central uncracked area of the samples; however we were not able to explain this feature.

\subsection{Crack growth results}

\subsubsection{Fully unloaded experiments}

Fig. 7 presents the experimental results for the fully unloaded experiments (four samples). First, Fig. 7(a) depicts the raw data. The curves can be separated into two parts: for low fracture energy, i.e. $\log G \leq 3.75$, the results depend on the mode I crack
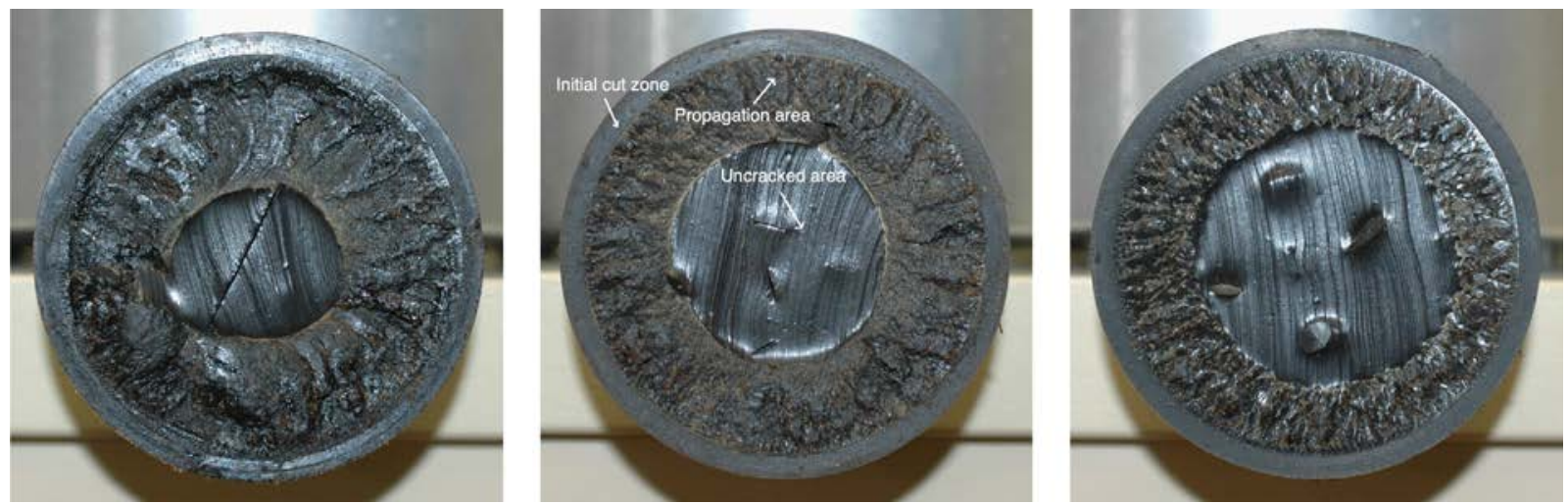

Fig. 6. Fracture surfaces for a given axial displacement. Left: $0.3 \mathrm{~mm}$ (sample FU3), middle: $0.5 \mathrm{~mm}$ (sample FU1), right: $1 \mathrm{~mm}$ (sample FU4). 

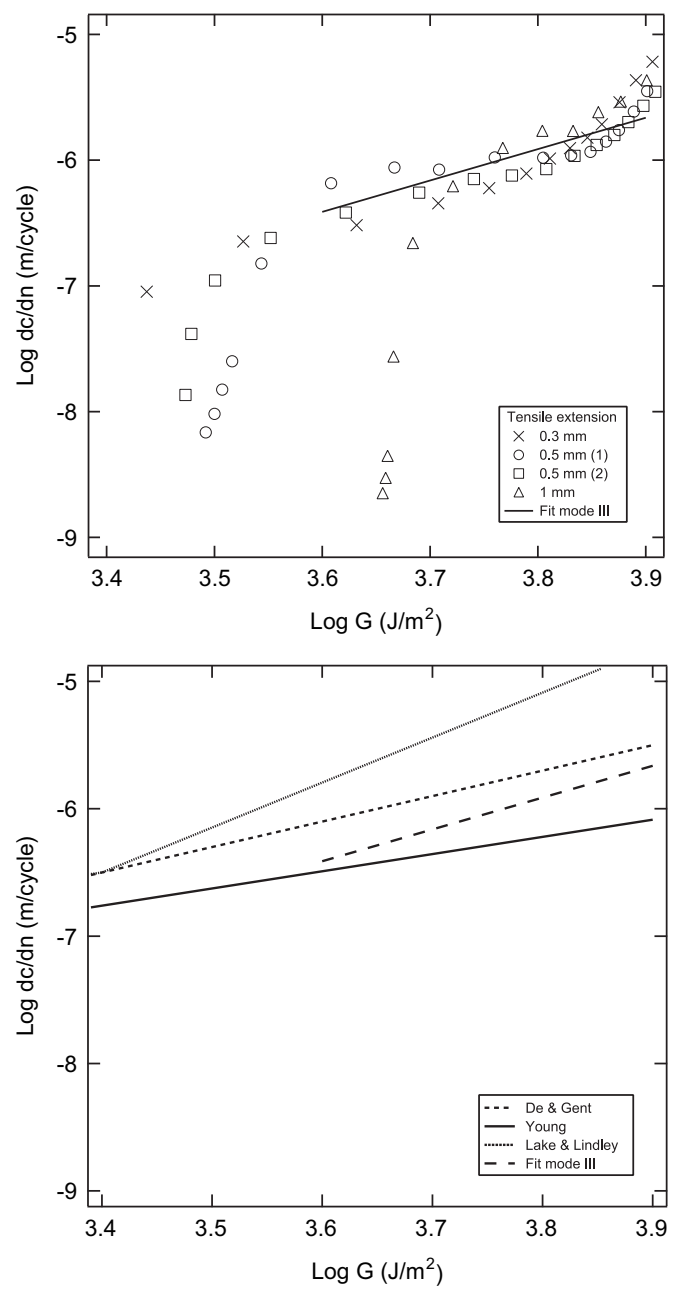

Fig. 7. Crack growth rate $d c / d n$ vs. fracture energy $G$ for unfilled NR vulcanizate under fully unloaded mode III loading conditions: (a, up) raw data, (b, down) fitted laws for high values of $G$ and comparison with published results for mode I.

opening, and for higher values of $G$ the four sets of experimental points coincide. So, one can argue that for low values of $G$ mode I highly influences the crack growth rate whereas for higher $G$ only mode III drives $d c / d n$. In order to quantitatively discuss these results, we adopt the approach of Lake and Lindley for mode I fatigue crack growth: they proposed to separate the $d c / d n$ vs. $G$ curves by the following set of equations (see Eqs. (2)-(5) in [2]):

$d c / d n=r, \quad G \leq G_{0}$

$d c / d n=A\left(G-G_{0}\right)+r, \quad G_{0} \leq G \leq G_{t}$

$d c / d n=B G^{\beta}, \quad G_{t} \leq G \leq G_{c}$

$d c / d n=\infty, \quad G=G_{c}$,

where $r, A, B$ and $\beta$ are the rate of growth constants, and $G_{0}, G_{t}$ and $G_{c}$ are the threshold fracture energies that separate the different crack growth regimes:

- Eq. (16) defines the minimum tearing energy for mechanooxidative crack growth $G_{0}$ under which there is no influence of $G$ on the crack growth rate,

- Eq. (17) stands for the linear relationship that correspond to low values of $G$ and ends for the threshold energy $G_{t}$, i.e. the fracture energy at which the dependence of crack growth rate on $G$ changes from linear to power law,
Table 3

Parameters of crack growth laws.

\begin{tabular}{lllll}
\hline Sample & $\begin{array}{l}\text { Axial } \\
\text { displacement }\end{array}$ & $\begin{array}{l}G_{0} \\
\left(\mathrm{~J} / \mathrm{m}^{2}\right)\end{array}$ & $\begin{array}{l}B \\
(\mathrm{~m} / \text { cycle }) /\left(\mathrm{J} / \mathrm{m}^{2}\right)\end{array}$ & $\beta$ \\
\hline FU3 & $0.3 \mathrm{~mm}$ & 1800 & $1.78 \times 10^{-15}$ & 2.33 \\
FU1 & $0.5 \mathrm{~mm}$ & 2950 & $1.78 \times 10^{-15}$ & 2.33 \\
FU2 & $0.5 \mathrm{~mm}$ & 2900 & $1.78 \times 10^{-15}$ & 2.33 \\
FU4 & $1 \mathrm{~mm}$ & 4500 & $1.78 \times 10^{-15}$ & 2.33 \\
De and Gent [8] & $0 \mathrm{~mm}$ & - & $3.81 \times 10^{-16}$ & 2.71 \\
Lake and Lindley [2] & - & 50 & $5 \times 10^{-14}$ & 2 \\
Young [3] & - & - & $4.46 \times 10^{-12}$ & 1.35 \\
\hline
\end{tabular}

- Eq. (18) represents the power law regime for high $G$ (note that in [2] the authors empirically set $\beta=2$ ),

- and finally Eq. (19) defines the critical fracture energy.

For the four experiments FU1-FU4, the parameter $G_{0}$ of the low $G$ regime (Eq. (17)) and the power law parameters of Eq. (18) are fitted; they are given in Table 3. These quantitative results strengthen the previous qualitative observations: mode I influences the low $G$ regime through $G_{0}$, but do not influence high $G$ regime for which the same values of $B$ and $\beta$ have been obtained.

Fatigue regime for low $G$, Eq. (17): The low $G$ regime is difficult to comment upon. Indeed, the value of $G_{0}$ determined by Lake and Lindley, i.e. $50 \mathrm{~J} / \mathrm{m}^{2}$, is highly inferior than our findings. To understand this discrepancy, our definition of $G_{0}$ must be clarified: here we define $G_{0}$ as the fracture energy for which the crack growth rate would be equal to $10^{-9} \mathrm{~m} /$ cycle if the low $G$ regime curve is extended to very low crack growth value. It is to note that in their paper, De and Gent did not measure this quantity [8].

In order to comment the difference between our values of $G_{0}$ and the one of Lake and Lindley, we invoke a study of Lindley who demonstrated that for mode I loading conditions with non-zero minimum force (or displacement), the crack growth rate decreases as the $R$-ratio, i.e. the ratio between the minimum and the maximum forces (or displacements), increases [14]; see also the survey of Mars and Fatemi for additional details [15]. During our experiments, the axial extension is prescribed and then it leads to small non-fully relaxing loading conditions. At the beginning of an experiment (high $G$, small crack length), the axial extension is very small as compared to the torsion strain. Therefore, the crack growth rate is unaffected by the axial extension. As the crack grows, the torsion strain diminishes, and $G$ decreases. When the torsion strain and the axial extension become the same order of magnitude, the effects of non-fully relaxing conditions emphasized above take place and the crack growth rate rapidly drops, as established by Lindley and Mars.

Fatigue regime for high $G$, Eq. (18): Now, we only focus on the fatigue regime for high $G$, in which there is no influence of mode I crack opening.

The present results in mode III given in Table 3 are compared with those of other authors also obtained for unfilled NR vulcanizates (obviously, some details of the chemical composition differ from one to the other): the results in mode III of De and Gent [8], the results in mode I of Lake and Lindley [2] obtained on thin strips with a small edge cut, and the results in mode I of Young [3] obtained with pure shear samples (at $50{ }^{\circ} \mathrm{C}$ and $40 \mathrm{~Hz}$ !). The corresponding values of the power law parameters of Eq. (18) are reported in Table 3. They were given in the corresponding papers for the two former sets of results, and recently calculated by Mars for the latter one [16]. The corresponding curves are plotted in Fig. 7(b). For high values of $G$, parameters and curves are similar, the major discrepancy is observed between our results and 


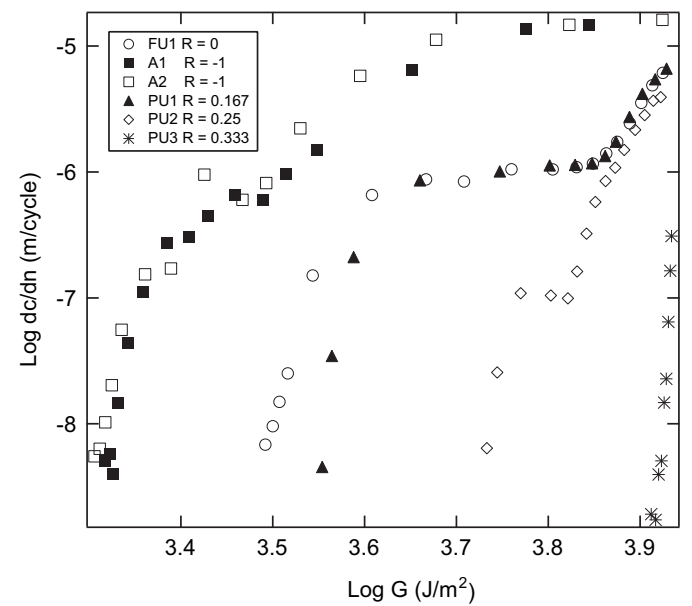

Fig. 8. Crack growth rate $d c / d n$ vs. fracture energy $G$ for unfilled NR vulcanizate under both partially unloaded and alternate mode III loading conditions.

the ones of De and Gent. Nevertheless, it must be noted that in this case the fitted parameters have been obtained only with two data points. The major conclusion is that for high values of $G$ crack growth rates in modes I and III are the same, and then can be determined with only one type of experiment.

\subsubsection{Other types of experiments}

To investigate the influence of non-relaxing loading conditions that has been commented in the previous remark, experiments with non-zero minimum torsion angle are conducted; they correspond to the samples named as PU in Table 2. Moreover, we took advantage of the versatility of the machine to perform alternate experiments; they correspond to samples A in Table 2. As this work is the first to propose experimental results for both partially unloaded and alternate mode III fatigue experiments in elastomers, the results cannot be compared with others. Indeed, the two types of experiment are considered simultaneously, and the results will be discussed as compared with the classical fully unloaded experiments described above and illustrated in the present section by the sample FU1.

Raw results are depicted in Fig. 8. The corresponding values of the parameters of the crack growth laws are given in Table 4. In Fig. 8, it is clear that the crack growth law is affected by the minimal angle. To discuss this influence, we define the torsion $R$-ratio $R^{*}$ by

$R^{*}=\frac{\phi_{\min }}{\phi_{\max }}$

In the present experiments, $R^{*}$ ranges from 0.167 to 0.333 for partially unloaded experiments, is equal to 0 for fully unloaded experiments, and is equal to -1 for alternate tests. For small positive values of $R^{*}$, i.e. sample PU1, the crack growth rate law is similar to the one in fully unloaded conditions $\left(R^{*}=0\right)$; only $G_{0}$ differs: it is higher when $R^{*}>0$. This difference is due to the dependence of $G_{0}$ on $R^{*}$ already exhibited in mode I for fully unloaded tests. For high values of $R^{*}$, i.e. samples PU2 and PU3, the slope of the law $\beta$ increases with $R^{*}: \beta=14$ for P2 and $\beta=100$ for P3. The crack growth rate is significantly lower than for FU1 and PU1. Moreover, $G_{0}$ also increases with $R^{*}$, which is in agreement with the results obtained for non-fully relaxing results in mode I [14]. Finally, we examine the alternate results. It is to be noted that these types of loading conditions are difficult to prescribe in mode I, and then the effect of such conditions on crack growth rate is unknown. As shown in Fig. 8, alternate loading conditions increase the crack growth rate by a factor 10 (for a given $G$ ) and reduces $G_{0}$
Table 4

Parameters of crack growth laws for partially unloaded and alternate loading conditions.

\begin{tabular}{lllc}
\hline Sample & $G_{0}\left(\mathrm{~J} / \mathrm{m}^{2}\right)$ & $B(\mathrm{~m} /$ cycle $) /\left(\mathrm{J} / \mathrm{m}^{2}\right)$ & $\beta$ \\
\hline A1/A2 & 2000 & $3.2 \times 10^{-15}$ & 2.5 \\
PU1 & 3500 & $1.78 \times 10^{-15}$ & 2.33 \\
PU2 & 5250 & - & 14 \\
PU3 & 8000 & - & 100 \\
FU1 & 3000 & $3.2 \times 10^{-15}$ & 2.33 \\
\hline
\end{tabular}

as compared to unloaded loading conditions. In fact, the decrease in $G_{0}$ is in continuity with the partially unloaded results: as $R^{*}$ increases (from -1 to 0.333 here), $G_{0}$ increases. Thus, we can argue that the fatigue crack growth in rubber depends on both the minimum and the maximum loading states.

\section{Conclusion}

In the present paper, the series of papers of Gent and coworkers dedicated to mode III fatigue of rubber has been revisited with the help of a modern multiaxial fatigue machine. A similar approach of the problem has been adopted: first a specific sample was designed and fabricated as in [8], the corresponding fracture energy was derived analytically as in [7], and numerically as in [9]. The experiments were conducted by taking advantage of the versatility of the machine: the application and control of a slight axial strain permits us to limit abrasion of cracked surface and the loading capabilities permit us to conduct fatigue tests with various loading ratios between -1 and 0.333 .

Some interesting results have been established. First, in regime of high $G$, modes I and III crack growth laws coincide. In the same regime, if we only consider results in mode III, small positive values of the $R$-ratio yield crack growth laws similar to $R^{*}=0$. Nevertheless, for some other values of this ratio, we observed important changes in the crack growth rate both for partially unloaded loading conditions with high minimum torsion angle $\left(R^{*}=0.333\right)$ and for alternate loading conditions $\left(R^{*}=-1\right)$. Second, in regime of low $G$ the crack growth rate laws in mode III highly depend on the loading conditions: the amount of mode I as well as the $R$-ratio substantially influence the critical threshold $G_{0}$.

\section{References}

1] C.L. Chow, W.H. Tai, C.T. Liu, Prediction of mixed-mode fracture in participate composite using a damage criterion, Tire Sci. Technol. 29 (2008) 79-90.

[2] G. Lake, P. Lindley, The mechanical fatigue limit for rubber, J. Appl. Polym. Sci. 9 (1965) 1233-1251.

[3] D.G. Young, Dynamic property and fatigue crack propagation research on tire sidewall and model compounds, Rubber Chem. Technol. 58 (1985) 785-805.

[4] E.E. Gdoutos, P.M. Schubel, I.M. Daniel, Determination of critical tearing energy of tyre rubber, Strain 40 (2004) 119-125.

[5] E. Verron, Study of the simple extension tear test sample for rubber with configurational mechanics, Int. J. Fract. 147 (2007) 219-225.

[6] D.W. Cha, H.Y. Jeong, Development of a mode III fatigue test method and a peel test method for tire specimens, Int. J. Fatigue 33 (2011) 912-920.

[7] H. Aboutorabi, T. Ebbot, A.N. Gent, O.H. Yeoh, Crack growth in twisted rubber disks. Part I: fracture energy calculation, Rubber Chem. Technol. 71 (1998) $76-83$.

[8] D.K. De, A.N. Gent, Crack growth in twisted rubber disks. Part II: experimental results, Rubber Chem. Technol. 71 (1998) 84-94.

[9] A.N. Gent, O.H. Yeoh, Crack growth in twisted rubber disks. Part III: effects of crack depth and location, Rubber Chem. Technol. 76 (2003) 1276-1289.

[10] M.W. Keller, S.R. White, N.R. Sottos, Torsion fatigue response of self-healing poly(dimethylsiloxane) elastomers, Polymer 49 (2008) 3136-3145.

[11] R.S. Rivlin, A.G. Thomas, Rupture of rubber. I. Characteristic energy for tearing, J. Polym. Sci. 10 (1953) 291-318. 
[12] A.E. Green, J.E. Adkins, Large Elastic Deformations, The Clarendon Press, Oxford, 1960.

[13] G. Marckmann, E. Verron, Comparison of hyperelastic models for rubberlike materials, Rubber Chem. Technol. 79 (2006) 835-858.

[14] P.B. Lindley, Relation between hysteresis and the dynamic crack growth resistance of natural rubber, Int. J. Fract. 9 (1973) 449-462.
[15] W.V. Mars, A. Fatemi, A literature survey on fatigue analysis approaches for rubber, Int. J. Fatigue 24 (2002) 949-961.

[16] W.V. Mars, A. Fatemi, A novel specimen for investigating the mechanical behavior of elastomers under multiaxial loading conditions, Exp. Mech. 44 (2004) 136-146. 